Bolm Inst. oceanogr., S Paulo, 31(2):101-104, 1982

\title{
SHORT-CUTS ON WAVE REFRACTION COMPUTATION
}

\author{
Alberto dos Santos FRANCO \\ IPT - Instituto de Pesquisas Tecnológicas do Estado de São Paulo S/A
}

\section{Synops is}

It is shown how to get $\mathrm{mid}$ of iterative process to compute wavelengths and how the celerities and their derivatives can be computed in terms of wavelengths for any depth, without using hyperbolic functions.

1 - Background

It is well known that the pressure wave records have a strong attenuation of the high frequency components of the wave train, and that such records can not be corrected in the time domain. In fact the Corrections would be feasible in the frequency domain, after the Fourier analysis of the pressure records, in terms of the frequency of the Fourier lines. Such corrections are usually computed iteratively because they are given in terms of the wavelength which is the independent variable in the frequency equation. This problem was solved using polynomials and we can show that a similar solution can be found to compute celerities.

According to the wave linear theory the celerity corresponding to a wave number $k$ and a depth $d$ is given by

$$
c=\sqrt{\frac{g}{k} \tanh (k d)} .
$$

In addition for deep seas

$$
c_{0}^{2}=g / k_{0}
$$

where

$$
\mathrm{k}_{0}=2 \pi / \mathrm{L}_{0}
$$

Lo being the deep-sea wave length. Thus, expression (1a) can be changed into

$$
\frac{c^{2}}{c_{0}^{2}} \frac{k}{k_{0}}=\tanh (k d) .
$$

Publ. n! 548 do Inst. oceanogr. da Usp.
Now

$$
c=\mathrm{L} / \mathrm{T}
$$

and

$$
c_{0}=L_{0} / T
$$

where $\mathrm{T}$ is the period considered as being constant; consequent1y,

$$
\frac{L^{2} k}{L_{0}^{2} k_{0}}=\tanh (k d) \text {. }
$$

Expression (1b) for $\mathrm{L}_{0}=\mathrm{L}$ and $\mathrm{k}_{0}=\mathrm{k}$ divided by (1b) gives

$$
\mathrm{L} / \mathrm{L}_{0}=\mathrm{k}_{0} / \mathrm{k} \text {, }
$$

hence the previous equation can be transformed into

$$
\frac{\mathrm{k}_{0}}{\mathrm{k}}=\tanh (\mathrm{kd})
$$

thus

$$
\mathrm{k}_{\mathrm{od}} \mathrm{d}=\mathrm{kd} \tanh (\mathrm{kd})
$$

or if we make

$$
\mathrm{k}_{0} \mathrm{~d}=\mathrm{z}_{0}
$$

and

$$
\mathrm{kd}=\underline{\mathrm{z}}
$$

we obtain

$$
z_{0}=z \tanh z \text {. }
$$

The main problem is now to solve this equation for $z$. Since the series development of tanh $z$ converges for 
$z<\pi / 2$ which corresponds to a relative depth $d / L \leq 1 / 4$, Elias (1971) had the idea of working out a series development to use it up to $d / L \leq 1 / 4$.

However if we plot the equation (1e) we see that for $z>1.36(d / L>0.21)$ the curve is nearly a straight line (Fig. 1). In the present paper, the author proposes to fitting a polynomial to the section of the curve between the abscissae 1.36 and 5.00 . Several polynomials were tried and it was concluded that a 4 th degree polynomial was the adequate one.

If the values of $\mathrm{z}$ and $\mathrm{z}_{0}$ are known it is easy to obtain any function of these elements. Value of $z$ obtained from iterative process and those computed with the two polynomials proved to be very close. The same technique can then be used in the computation of wave refraction were the celerities and their derivatives can be expressed in terms of $\mathrm{z}$ and $\mathrm{z}_{0}$ only.

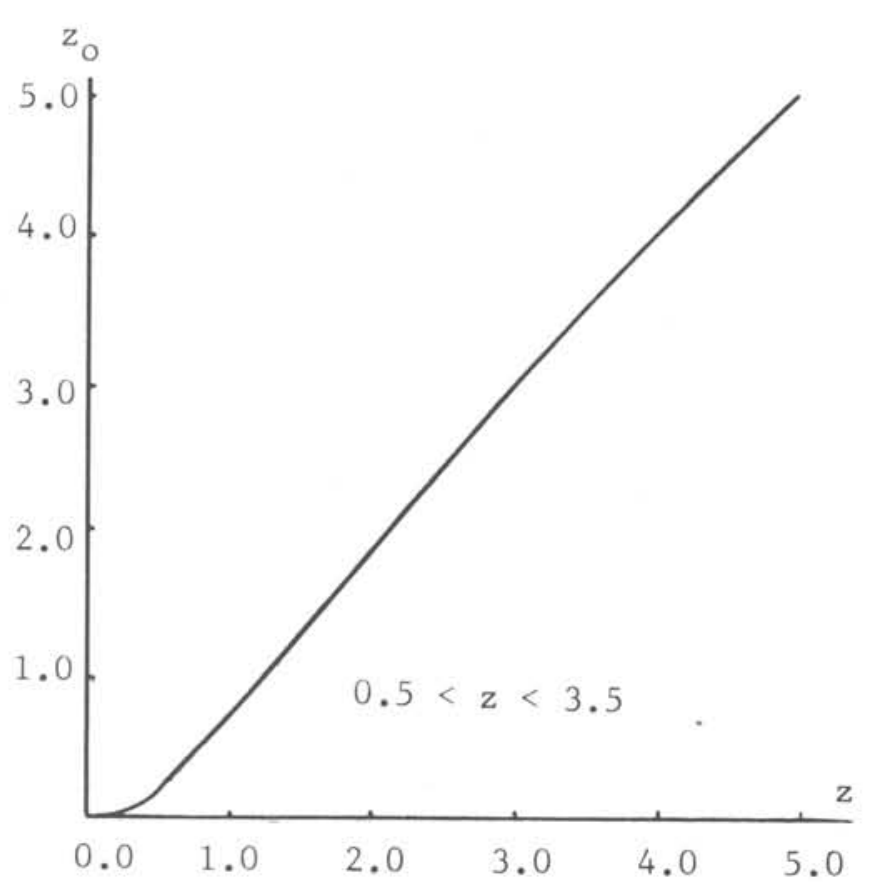

Fig. 1. Function $z_{0}=z \tanh z$.

2 - Finding $z$

Let us develop (1e) $\mathrm{z}_{0}=\mathrm{z}_{0}(\mathrm{z})$ according to the powers of $\mathrm{z}$ :

$z_{0}=z\left(z+a_{1} z^{3}+a_{2} z^{5}+a_{3} z^{7}\right)$

where

$a_{1}=-1 / 3, a_{2}=2 / 15$ and $a_{3}=-17 / 315$
We derive from (2a)

$$
\begin{aligned}
z^{2} & =z_{0} /\left(1+a_{1} z^{2}+a_{2} z^{4}+a_{1} z^{6}\right) \\
& \simeq z_{0}\left(1-\varepsilon+\varepsilon^{2}-\varepsilon^{3}\right)
\end{aligned}
$$

where

$$
\varepsilon=a_{1} z^{2}+a_{2} z^{4}+a_{2} z^{6}
$$

If we use this value of $\varepsilon$ in the preceding expression and neglect the powers of $z$ higher than the $6^{\text {th }}$ we obtain:

$$
\begin{array}{r}
z^{2}=z_{0}\left[1-a_{1} z^{2}+\left(a_{1}^{2}-a_{2}\right) z^{4}-\right. \\
\left.\left(a_{3}+2 a_{1} a_{2}+a_{1}^{3}\right) z^{6}\right]
\end{array}
$$

or

$z^{2}=z_{0}\left(1+A_{1} z^{2}+A_{2} z^{4}+A_{3} z^{6}\right)$

where

$$
\begin{aligned}
& A_{1}=-a_{1}=1 / 3 \\
& A_{2}=a_{1}^{2}-a_{2}=-1 / 45
\end{aligned}
$$

and

$$
A_{3}=-\left(a_{1}+2 a_{1} a_{2}+a_{1}^{3}\right)=2 / 945
$$

Let us now solve (2c) for $z$. If we assume that the solution must be compatible with $(2 \mathrm{c})$ we can write

$$
z^{2}=z_{0}\left(1+B_{1} z_{0}+B_{2} z_{0}^{2}+B_{3} z_{0}^{3}\right)
$$

where $B_{i}$ can be found as follows. If we use this value of $z^{2}$ in the right hand side of $(2 c)$ and neglect powers of $z_{0}$ larger than the fourth we have

$$
\begin{gathered}
z^{2}=z_{0}\left[1+A_{1} z_{0}\left(1+B_{1} z_{0}+B_{2} z_{0}^{2}\right)+A_{2} z_{0}^{2} \times\right. \\
\left.x\left(1+2 B_{1} z_{0}\right)-A_{3} z_{0}^{3}\right]= \\
=z_{0}\left[1+A_{1} z_{0}+\left(A_{1} B_{1}+A_{2}\right) z_{0}^{2}+\right. \\
\left.\left(A_{1} B_{2}+2 A_{2} B_{1}+A_{2}\right) z_{0}^{3}\right]
\end{gathered}
$$

Equating the coefficients of the equal powers of $z_{0}$ in (2e) and (2f) and solving for $\mathrm{B}_{i}$ we find

$$
\begin{aligned}
& \mathrm{B}_{1}=\mathrm{A}_{1}=1 / 3 \\
& \mathrm{~B}_{2}=\mathrm{A}_{1} \mathrm{~B}_{1}+\mathrm{A}_{2}=4 / 45 \\
& \mathrm{~B}_{3}=\mathrm{A}_{1} \mathrm{~B}_{2}+2 \mathrm{~A}_{2} \mathrm{~B}_{1}+\mathrm{A}_{3}=16 / 945
\end{aligned}
$$


These are the coefficients of $(2 e)$, which are very accurate if $z_{0}$ does not exceed 1.35772. For $1.35772 \leq \mathrm{z} \leq$ 4.99954 we tried polynomials from the second to the fourth degree, the latter being the best fitted:

$$
\begin{aligned}
z= & 0.53753+0.54931 z_{0}+0.14440 z_{0}^{2}- \\
& 0.02085 z_{0}^{3}+0.00114 z_{0}^{4} \quad(2 h)
\end{aligned}
$$

For $z_{0} \geq 5$ we can assume that $z=z_{0}$

3-Finding the celerity and $i$ ts derivatives Since $\omega=c k$ expression ( $1 a$ ) can be changed into:

$$
c=\frac{g}{c k} \tanh z=f(z)
$$

or according to (1e)

$$
c=\frac{g z_{0}}{\omega z}-=f(z)
$$

Using the subscript $(i, j)$ to denote the derivatives with respect to $x$ or $y$ and $(i j)$ the derivative with respect to $x$ and $y$ we obtain from ( $3 a$ )

$$
\begin{aligned}
& c_{i, j}=f^{\prime}(z) z_{i, j} \\
& c_{i j}=f^{\prime}(z) z_{i j}+f^{\prime \prime}(z) z_{i} z_{j}
\end{aligned}
$$

where

$$
f^{\prime}(z)=\frac{g}{\omega} \operatorname{sech}^{2} z
$$

and

$$
f^{\prime \prime}(z)=-\frac{g}{\omega} \operatorname{sech}^{2} z \tanh z
$$

Using (1e) to express $z_{i}, z_{j}$ and $z_{i j}$ in terms of $\left(z_{0}\right)_{i},\left(z_{0}\right)_{j}$ and $\left(z_{0}\right)_{i j}$ wē have after algebraic strait forward manipulations:

$$
c_{i, j}=\frac{g}{\omega} \frac{1}{A+z}\left(z_{0}\right)_{i, j}
$$

and

$$
\begin{aligned}
& c_{i j}=\frac{g}{\omega}\left\{\frac{1}{A+Z}\left(z_{0}\right)_{i j}-\right. \\
& \left.\frac{2 A\left(z+z_{0}+A z_{0}-z_{0}\right)}{z_{0}(A+z)^{3}} \times\left(z_{0}\right)_{i}\left(z_{0}\right)_{j}\right\}
\end{aligned}
$$

where

$$
A=\frac{1}{2} \sinh 2 z=\tanh z /\left(1-\tanh ^{2} z\right)
$$

or, according to (1b),

$$
A=z_{0} /\left(z^{2}-z_{0}^{2}\right)
$$

But we have from (1a)

$$
\left(z_{0}\right)_{i, j}=k_{0} d_{i, j}
$$

and

$$
\left(z_{0}\right)_{i j}=k_{0} d_{i j}
$$

hence we can change $(3 g)$ and $(3 h)$, respectively, into

$$
c_{i, j}=\frac{k_{0} g}{\omega} \frac{1}{A+z} d_{i, j}
$$

and

$$
\begin{aligned}
c_{i j}= & \frac{k_{0} g}{\omega}\left\{\frac{1}{A+z} d_{i j}-\right. \\
& \left.\frac{2 k_{0} A\left[z+z_{0}(A+z)-z_{0}\right]}{z_{0}(A+z)^{3}} d_{i} d_{j}\right\}
\end{aligned}
$$

\section{4 - Conclusion}

The Wilson (1966)'s method to compute wave refraction can be improved by introducing the above formulation for computing wavelengths, celerities and derivatives of wave celerities. If so, the computer's time is reduced by a factor of two.

\section{Acknowledgements}

I am very much indebted to $\mathrm{Mr}$. Waldemar Tavares $\mathrm{Jr}$. for introducing the proposed modifications in the original program and testing the results and to Mr. José Maria Segovia Puyol for helping in the algebraic manipulations.

\section{References}

ELIAS, N. T. P. 1971 Sobre o cá1culo do comprimento de onda na teoria 1 i- 
near. Simpósio sobre dados de agitação marítima. Laboratório Nacional de Engenharia Civil - LNEC/Lisboa.

WILSON, W. S. 1966. A method for calculating and plotting surface wave rays. Tech. Memo. coast. Engng Res. Cent. U.S., (17):1-57.

(Manuscript received on 04/Mar./1982; accepted on 28/Sep./1982) 